\title{
Pretreatment heterogeneous enhancement pattern of hepatocellular carcinoma can be new predictor for early response to lenvatinib
}

Yusuke Kawamura ( $\nabla$ k-yusuke@toranomon.gr.jp )

Toranomon Hospital https://orcid.org/0000-0001-6148-0042

Masahiro Kobayashi

Toranomon Hospital

Junichi Shindoh

Toranomon Hospital

Yuta Kobayashi

Toranomon Hospital

Kayoko Kasuya

Toranomon Hospital

Tomoya Sano

Toranomon Hospital

Shunichiro Fujiyama

Toranomon Hospital

Tetsuya Hosaka

Toranomon Hospital

Satoshi Saitoh

Toranomon Hospital

Hitomi Sezaki

Toranomon Hospital

Norio Akuta

Toranomon Hospital

Fumitaka Suzuki

Toranomon Hospital

Yoshiyuki Suzuki

Toranomon Hospital

Kenji Ikeda

Toranomon Hospital

Yasuji Arase

Toranomon Hospital

Masaji Hashimoto 
Toranomon Hospital

\section{Hiromitsu Kumada}

Toranomon Hospital

\section{Research article}

Keywords: lenvatinib; hepatocellular carcinoma; computed tomography; positron emission tomography; unresectable hepatocellular carcinoma

Posted Date: July 3rd, 2019

DOI: https://doi.org/10.21203/rs.2.10911/v1

License: (9) This work is licensed under a Creative Commons Attribution 4.0 International License. Read Full License 


\section{Abstract}

Background: The aims of this study were to identify useful predictors of early tumor response to lenvatinib, and to evaluate the utility of estimation of tumor differentiation from pretreatment image analysis. Methods: We evaluated 37 consecutive patients with unresectable hepatocellular carcinoma (HCC) diagnosed by dynamic computed tomography (CT) who received lenvatinib. Pretreatment arterialand portal-phase dynamic CT images were classified into three enhancement patterns: Type- 2 is a homogeneous enhancement pattern with increased arterial blood flow; Type- 3 is a heterogeneous enhancement pattern with a septum-like structure; and Type-4 is a heterogeneous enhancement pattern with irregularly shaped ring structures. Generally, macroscopic classification of the nodular type of SNEG and CMN types strongly relates to the Type-3 enhancement pattern, and histologically, the Type- 1 enhancement pattern represents well-differentiated HCC, while the Type-2 and - 3 patterns represent moderately-differentiated HCC; the Type-4 enhancement pattern is a significantly specific feature for predicting poorly-differentiated HCC. Treatment response was evaluated using mRECIST at 8-12 weeks after initiation of lenvatinib. Results: In early treatment response evaluation, 6 of 37 patients $(16 \%)$ achieved a complete response (CR), 22 (59\%) experienced a partial response (PR), 6 (16\%) had stable disease (SD), and $3(8 \%)$ had progressive disease (PD); therefore, 28 of 37 patients (76\%) experienced an objective response (OR). By dynamic CT enhancement pattern using mRECIST, the objective response rate (ORR) was significantly elevated along with increasing heterogeneity of enhancement pattern from Type-2 (54\%) to Type-4 pattern (89\%; $\mathrm{P}=0.046)$. Multivariate logistic regression analysis revealed that a pretreatment dynamic CT heterogeneous enhancement pattern (Type-3 and -4) (hazard ratio, 6.12; $\mathrm{P}=0.040$ ) is a useful predictor of early OR. Conclusion: Lenvatinib provided a good early treatment response in patients with unresectable HCC. Estimation of tumor differentiation using image analysis was also useful for predicting early tumor response.

\section{Background}

Hepatocellular carcinoma (HCC) is the most common type of liver cancer, which is the third leading cause of cancer[1]. For HCC, the Barcelona Clinic Liver Cancer (BCLC) staging system is currently the most widely used[2, 3]. BCLC intermediate-stage disease may be further subclassified based on the Up-to-7 criteria[4] and liver function using the Child-Pugh system[5]. In patients with tumors within the Up-to-7 criteria and with good liver function, transarterial chemoembolization (TACE) is recommended. Previously, many chemotherapeutic agents were used for chemoembolization, and TACE was repeatedly performed until treatment could no longer be administered. Although responses may improve if different agents are used (especially cisplatin[6, 7]), TACE efficacy is limited. As a result of repeated TACE, many patients become TACE-refractory, and liver function declines[8]. About 10 years ago, the molecular targeted agent sorafenib became available for the treatment of unresectable HCC $[9,10]$, and worldwide, HCC treatment trended toward switching from TACE to sorafenib before liver function decline[11-14]. The OPTIMIS study[14] also suggested that switching from intraarterial treatment to sorafenib could extend survival. However, the efficacy of sorafenib in this setting is modest: median overall survival (OS) is $<1$ year and 
the objective response rate (ORR) is $<5 \%$. Thus, there remains a critical and unmet need for aggressive development of new and more effective agents for advanced HCC.

Recently, prior to approval elsewhere in the world, lenvatinib became available as a new molecular targeted agent for the first-line treatment of unresectable advanced HCC in Japan[15]. Lenvatinib, an inhibitor of vascular endothelial growth factor receptor (VEGFR) 1-3, fibroblast growth factor receptor (FGFR) 1-4, platelet-derived growth factor receptor (PDGFR)-a, Ret, and Kit, was reported to be noninferior to sorafenib with respect to OS in patients with untreated advanced HCC[16]. It was the second molecular targeted agent to demonstrate efficacy as a first-line treatment for unresectable HCC; lenvatinib treatment results in a higher ORR compared to sorafenib ( $18.8 \%$ vs $6.5 \%$, respectively, as evaluated by RECIST 1.1)[16]. In several treatment algorithms [2, 3], only the presence or absence of tumor hypervascularity on contrast enhancement study has been discussed for optimizing selection of treatment for HCC, without evaluation of the malignant potential of target nodules. We previously reported that a "heterogeneous enhancement pattern with a septum-like structure" in the arterial phase of dynamic computed tomography (CT) analysis accurately predicts macroscopic classification of the nodular types[17] of simple nodular type with extranodular growth (SNEG) and confluent multi-nodular (CMN) type of HCC, and that a "heterogeneous enhancement pattern with irregular ring-like structures" in the arterial phase of dynamic CT accurately predicts the histopathological grade of poorly-differentiated HCCs; we named these enhancement patterns "Type-3" and "Type-4," respectively.[18]

These unique enhancement patterns are also correlated with oncological aggressiveness of HCC [19] and they may guide an optimal approach in ablation therapy [20]. Given the potential ability of the enhancement patterns of HCC in predicting malignant potential of tumor and response to treatments, we sought to investigate the powers of these unique radiological enhancement patterns of HCC as a new pretreatment surrogate marker for predicting the response to lenvatinib.

\section{Methods}

Study population

From April 2018 to February 2019, 68 patients received systemic anticancer treatment using lenvatinib for unresectable HCC at the Department of Hepatology, Toranomon Hospital, Tokyo, Japan. In this study, the following inclusion criteria were used: 1) triple-phase dynamic CT study performed prior to initiation of lenvatinib, 2) tumor with hyperenhancement in the arterial phase of dynamic CT, 3) performance of triple-dynamic CT to evaluate treatment response 8-12 weeks after initiation of lenvatinib, 4) Child-Pugh class A liver function at the time of lenvatinib initiation, 5) BCLC stage A to $C$ tumor(s), 6) unresectable $\mathrm{HCC}$ and patient does not want to undergo local ablation or chemoembolization therapy for various reasons (i.e., tumor size, number and location, extrahepatic metastasis, TACE refractoriness, and various complications), 7) no treatment history of lenvatinib, 8) at least one measurable target nodule in the liver, 9) treatment interval of $>28$ days since previous tyrosine kinase inhibitor (TKl; sorafenib or regorafenib) therapy, and 10) an observation period of ${ }^{3} 8$ weeks. Finally, 37 patients met these inclusion criteria. All 
procedures followed were in accordance with the ethical standards of the responsible committees on human experimentation (institutional and national) and with the Helsinki Declaration of 1975. And, the study was approved by the Institutional Review Board of our hospital (protocol number; 1438-H/B).

Contrast infusion and CT protocol

All patients underwent triple-phase dynamic CT. CT was performed with a 64-multidetector CT (MDCT) scanner (Aquilion 64, Canon Medical Systems, Tochigi, Japan) with the following scanning parameters: rotation time, 0.5 seconds; beam collimation, $64 \times 0.5 \mathrm{~mm}$; section thickness and interval, $5 \mathrm{~mm}$; beam pitch, 0.83; tube voltage, $120 \mathrm{kV}$; and tube current, $150 \mathrm{mAs}$. All helical scans were started at the top of the liver and proceeded in a cephalocaudal direction. Unenhanced and three-phase contrast-enhanced helical scans of the whole liver were acquired. Patients were instructed to hold their breath with exhalation during scanning. An automatic bolus-tracking program (Sure Start, Canon Medical Systems) was used to time the start of acquisition in each phase after contrast injection (nonionic contrast material with an iodine concentration of $350 \mathrm{mg} \mathrm{l} / \mathrm{mL}$ iomeprol [Iomeron 350, Eisai, Tokyo] at a dose of 100-120 $\mathrm{mL} /$ body). Attenuation at the axis of the celiac artery level was monitored by one radiology technician; the region-of-interest (ROI) cursor $\left(1 \mathrm{~cm}^{2}\right)$ was placed in the abdominal aorta. Real-time low-dose $(120 \mathrm{kV}$, $25 \mathrm{mAs}$ ) serial monitoring studies were begun 5 seconds after the start of contrast injection. The trigger threshold level was set at 100 Hounsfield units (HUs). Double arterial phase acquisition was started 15 and 20 seconds after triggering, and portal phase and delayed phase acquisition were started 70 and 180 seconds after the start of the contrast injection, respectively.

Diagnosis of HCC

The diagnosis of HCC was based predominantly on image analysis using dynamic CT. When a liver nodule showed hyperattenuation in the arterial phase of the dynamic study and washout in the portal or delayed phase, the nodule was diagnosed as HCC.

Imaging analysis of HCC and definitions of dynamic CT enhancement patterns

Before treatment, the dynamic study enhancement pattern on the arterial and portal phases was classified into one of four types defined in our previous report.[18] The Type-1 pattern represented a homogeneous enhancement pattern with no increase in arterial blood flow, and the entire image was uniform during the arterial and portal phases. The Type-2 pattern represented a homogeneous enhancement pattern with increased arterial blood flow, and the entire image was uniform during the arterial and portal phases. The Type-3 pattern represented a heterogeneous enhancement pattern with septations, with heterogeneous enhancement and septations in the arterial phase, while the septations 
resembled a near-uniform tumor tissue periphery in the portal phase. The Type- 4 pattern represented a heterogeneous enhancement pattern with irregular ring-like structures; the arterial phase was marked by the presence of irregularly shaped ring areas of enhancement and areas of little blood flow relative to the periphery of the tumor tissue, and the portal phase was characterized by areas of reduced blood flow (Figure 1).

The enhancement pattern on the arterial and portal phases of dynamic CT was determined by three expert hepatologists. Generally, macroscopic classification of the nodular type of SNEG and CMN types strongly relates to the Type-3 enhancement pattern, and histologically, the Type-1 enhancement pattern represents well-differentiated HCC, while the Type-2 and -3 patterns represent moderately-differentiated HCC; the Type- 4 enhancement pattern is a significantly specific feature for predicting poorly-differentiated HCC.[18]

In this study, all target HCC nodules appeared to be hypervascular; therefore, we classified all nodules into three enhancement patterns (Type-2 to -4 ). The enhancement pattern that accounts for $70 \%$ of the nodule is defined as the predominant enhancement pattern.

In addition, to analyze mean $\mathrm{HU}$ (HU-mean) of intrahepatic target tumors, a circular ROI was drawn on the axial plane to include the largest surface of the target lesion, and the HU-mean in each tumor was calculated.[21] The hepatic HU-mean was measured in as many of the four segments (lateral, medial, anterior, and posterior) as possible at the level of the main portal vein, with an ROI of approximately 200 $\mathrm{mm}^{2}$.[22] Finally, we calculated the median tumor-to-liver ratio (TLR) for each target tumor.

Imaging analysis of HCC using FDG-PET/CT

Within 1 month before initiation of lenvatinib, fluorine-18-fluorodeoxyglucose (FDG)-positron emission tomography (PET) /CT was performed with a dedicated whole-body PET scanner (Biograph mCT Flow40, Siemens Healthcare, Germany). Using a software for semi-quantitative analysis (SYNAPSE VINCENT ver.4, FUJIFILM MEDICAL SYSTEMS, Japan), the volume of interest (VOI) was drawn along the outline of the tumor and the maximum SUV (SUV-max) and mean SUV (SUV-mean) in each intrahepatic target tumor were calculated. After that, to measure normal liver activity, three non-overlapping spherical $1 \mathrm{~cm}^{3}$-sized VOls were drawn in the liver (two in the right lobe and one in the left lobe) on the axial PET images, avoiding the areas of the HCC seen on

dynamic CT. The TLR was calculated using the following equation: TLR = SUV-max of the tumor/SUVmean of the normal liver.

In 37 patients enrolled in this study, 17 patients underwent PET/CT before initiation of lenvatinib. We selected TLR $\geq 2$ to indicate high malignant potential based on previous reports.[23-25] 
Lenvatinib (Eisai, Tokyo, Japan) was given orally to the majority of patients at either $8 \mathrm{mg} / \mathrm{day}$ for patients $<60 \mathrm{~kg}$ or $12 \mathrm{mg} /$ day for patients $\geq 60 \mathrm{~kg}$; treatment was discontinued when any unacceptable or serious adverse events (AEs) or significantly clinical tumor progression were observed. According to the guidelines for administration of lenvatinib, the drug dose should be reduced or the treatment interrupted when a patient develops grade $\geq 3$ severe $A E s$ or any unacceptable grade 2 drug-related AEs occur. AEs were assessed using the National Cancer Institute's Common Terminology Criteria for Adverse Events (CTCAE), version 4.0.[26] If a drug-related AE occurred, dose reduction or temporary interruption was maintained until the symptom was resolved to Grade 1 or 2 , according to the guidelines provided by the manufacturer.

Treatment response evaluation

Treatment response was evaluated in accordance with mRECIST.[27] The liver was examined by dynamic CT. Tumor assessments were generally performed every 4-8 weeks.

\section{Definition of TACE failure/refractoriness}

TACE failure was defined as an insufficient response after $\geq 2$ consecutive TACE procedures as evident on response evaluation $\mathrm{CT}$ or magnetic resonance imaging (MRI) after 1-3 months, even after the chemotherapeutic agent was changed and/or the feeding artery was reanalyzed. In addition, the appearance of a higher number of lesions in the liver than that recorded at the previous TACE procedure (other than the nodule being treated) was added to the definition of TACE failure/refractoriness.[28]

Follow-up protocol

Physicians examined patients every 1-2 weeks after initiation of lenvatinib, and biochemical laboratory and urine tests were also performed. After initiation of lenvatinib, patients underwent dynamic CT to evaluate early treatment response at 8-12 weeks. After the first evaluation of treatment response, dynamic CT was performed every 2-3 months.

Statistical analysis

Data are expressed as the median and range. Differences in background features between each parameter were analyzed by the chi-squared test, Fisher's exact test, Mann-Whitney U test, and Kuraskal- 
Wallis test. Significance of trends in treatment response evaluated using mRECIST (OR vs. non-OR) and dynamic CT enhancement patterns was determined with the Cochran-Armitage trend test. Independent factors associated with treatment response were studied using multivariate logistic regression analysis. Potential prognostic factors for OR after initiation of lenvatinib included the following 17 variables: gender, age, body mass index, etiology of background liver disease, platelet count, serum albumin, bilirubin, prothrombin activity, aspartate aminotransferase (AST), alpha-fetoprotein (AFP), des-g-carboxy prothrombin (DCP), tumor diameter, number, macrovascular invasion, extrahepatic metastasis, dynamic study enhancement pattern (for a few cases, Type-3 and -4 are collectively referred to as a heterogeneous enhancement pattern), and initial dose of lenvatinib. In this multivariate analysis, to detect true factors, the integrated score was excluded.

Several variables were transformed into categorical data consisting of two simple ordinal numbers for univariate and multivariate analyses. All factors that were at least marginally associated with $\mathrm{OR}$ $(P<0.15)$ in univariate analysis were entered into a multivariate logistic regression analysis. Significant variables were selected by a stepwise method. A two-tailed $P$-value less than 0.05 was considered to be statistically significant. Data analysis was performed with the Statistical Package for Social Sciences (SPSS) version 16.0 (SPSS Inc, Chicago, IL).

\section{Results}

\section{Clinical profiles and laboratory data}

Table 1 summarizes the clinical profile and laboratory data of $37 \mathrm{HCC}$ patients treated with lenvatinib in this study. The male:female ratio was 2.36:1. Hepatitis $\mathrm{C}$ virus (HCV) antibody was detected in $62.1 \%$ of patients. Overall, 29 patients $(78 \%)$ received an initial dose of lenvatinib according to body weight. Based on pretreatment image analysis, the median tumor diameter was $31 \mathrm{~mm}, 18$ of 37 patients (49\%) met the Up-to-7 criteria, and 16 of 37 patients (43\%) presented with BCLC stage $C$ disease; 6 of these 16 patients (38\%) presented with portal vein invasion ( $V p 2, n=4 ; V p 3$ and $V v 3, n=1$; and $V p 4, n=1)$ and 14 of 16 patients (88\%) presented with extrahepatic metastasis. In addition, 4 patients $(11 \%)$ had a history of treatment with other TKIs, and 33 patients (89\%) had a TACE failure/refractoriness status. Median levels of AFP and DCP were $127.4 \mathrm{mg} / \mathrm{L}$ and $277.0 \mathrm{AU} / \mathrm{L}$, respectively. With respect to pretreatment dynamic CT enhancement pattern, 13 patients (35\%) had the Type-2 pattern, 15 patients (41\%) had the Type-3 pattern, and 9 patients (24\%) had the Type-4 pattern. The median observation period was 6.6 months. Optional data about the relative dose intensity (RDI) of lenvatinib were expressed as the median (range), as follows: $74 \%(30-150 \%)$ at 8 weeks and $69 \%(31-138 \%)$ at 12 weeks.

\section{Evaluation of treatment response to lenvatinib}

In early treatment response evaluation using mRECIST, 6 of 37 patients (16\%) experienced a complete response (CR), 22 (59\%) experienced a partial response (PR), 6 (16\%) had stable disease (SD), and 3 (8\%) 
had progressive disease (PD); therefore, 28 of 37 patients (76\%) experienced an OR. By BCLC stage (A or B vs. C), the ORR was $67 \%$ for patients with BCLC stage A or B disease and $88 \%$ for patients with BCLC stage $C$ disease (Table 2). TACE failure/refractoriness was present in 20 of 21 patients (95\%) with BCLC stage $B$ disease, and 13 of 16 (81\%) with BCLC stage $C$ disease. Among patients with BCLC stage $C$ disease, 14 of $16(88 \%)$ patients had extrahepatic spread; however, an interventional treatment approach was enforced for an extrahepatic metastatic lesion in only 1 patient $(7 \%)$.

\section{Evaluation of treatment response after initiation of lenvatinib by dynamic CT enhancement pattern (Type- 2 to -4$)$}

In the early treatment response evaluation by dynamic CT enhancement pattern using mRECIST, ORR was significantly elevated along with increasing heterogeneity of enhancement pattern from Type-2 (54\%) to Type-4 pattern ( $89 \% ; P=0.046)$. Target nodule (HU) and TLR values were significantly different between enhancement patterns $(P=0.020$ and $P=0.015$, respectively); nodules with a Type-4 enhancement pattern had the lowest median target nodule $(\mathrm{HU})(67.67 \mathrm{HU})$ and TLR (1.00), respectively (Talbe 3).

\section{Predictor of OR to lenvatinib from initial clinical information}

Multivariate logistic regression analysis revealed one independent predictive factors for early OR after initiation of lenvatinib: a heterogeneous enhancement pattern (Type-3 and -4 ) on pretreatment dynamic CT (hazard ratio [HR], 6.12; 95\% confidence interval [Cl], 1.09-34.46; $P=0.040$ ) (Table 4).

\section{Relationship between dynamic CT enhancement pattern and TLR using FDG-PET in tumor response evaluation}

In this study, 17 of 37 patients (46\%) underwent FDG-PET. Although only a small number of cases were examined, setting a TLR of 2.0 as a cut-off for HCC of highly malignant potential revealed that in the TLR $\geq 2.0$ group, all cases had a heterogeneous enhancement pattern, and all cases achieved an OR early in treatment. In contrast, in the TLR $<2.0$ group, 5 of 13 patients (38\%) had a heterogeneous enhancement pattern, and the ORR was 69\%. (Table 5)

\section{Frequency of Grade ${ }^{33}$ AEs following initiation of lenvatinib}

No Grade 4 AEs were observed during the observation period. With respect to Grade 3 AEs, 5 of 37 patients (14\%) experienced hypertension, 3 of 37 (8\%) experienced hand foot skin reaction, 3 of 37 (8\%) experienced appetite loss, 2 of 37 (5\%) experienced diarrhea, 9 of 37 (24\%) experienced decreased 
platelets counts, 2 of 37 (5\%) experienced elevated AST, 5 of 37 (14\%) experienced elevated total bilirubin, 5 of $37(14 \%)$ experienced hepatic encephalopathy, and 2 of 37 (5\%) experienced elevated urinary protein.

\section{Discussion}

Patients with unresectable HCC who show intolerability to sorafenib or failure of another TKI need second- and third-line treatment options. Lenvatinib, a newer TKI[15, 16], has become available as a firstline agent for unresectable HCC in Japan. Lenvatinib demonstrated efficacy as a first-line treatment for unresectable HCC, and is associated with a higher ORR compared with sorafenib[16]. In this study, the overall ORR was similar to that observed in a previous Phase III study. By BCLC clinical stage, the ORR was $67 \%$ among patients with stage $A$ or $B$ disease, while it was $88 \%$ in patients with stage $C$ disease. An explanation for this result is that in this cohort, metastatic lesions, particularly peritoneal dissemination, lymph node metastases, and adrenal grand metastases, responded relatively well compared with intrahepatic target nodules. Therefore, patients with BCLC stage $C$ disease experienced a relatively high response compared with BCLC stage A or B patients. The majority of BCLC stage A or B HCC patients (95\%) presented with TACE failure/refractoriness at the time of lenvatinib initiation. However, although 14 of $16(88 \%)$ BCLC stage $C$ patients had extrahepatic spread, an interventional treatment approach was directed against extrahepatic metastatic lesions in only 1 case ( $7 \%)$. Therefore, it can be assumed that the other metastatic lesions were intervention-naïve nodules. Such a difference between groups might have influenced treatment outcomes.

In addition, a second aim was to evaluate lenvatinib treatment response by dynamic CT enhancement pattern. HCCs have very heterogeneous tumor characteristics, even in the same patient. Notably, in patients with advanced HCC, we frequently encounter several tumors and various enhancement patterns within a single patient's liver. Therefore, it is difficult to select the best overall treatment method and chemotherapeutic agent by sampling just one of many tumors. Moreover, tumor biopsies are not easy to perform in all cases for several reasons, including tumor localization, risk of tumor dissemination, and risk of bleeding due to liver cirrhosis.

In these situations, we previously reported that a Type-3 enhancement pattern accurately predicts macroscopic classification of the nodular type of SNEG and CMN types of HCC and that a Type-4 enhancement pattern accurately predicts the histopathological grade of poorly-differentiated HCC.[18] Our past work revealed a strong relationship between the Type-4 enhancement pattern and HCC recurrence characterized by multiple nodules and/or portal vein invasion following RFA.[19]

However, the utility of these dynamic CT enhancement patterns in predicting response to TKIs, especially lenvatinib, has not been clear until now. In this study, a heterogeneous enhancement pattern (Type-3 and -4) on dynamic CT was a significant pretreatment predictor of OR following initiation of lenvatinib (Figure 2). In addition, the ORR was significantly higher for Type-4 lesions compared to Type-2 lesions. Although these are preliminary data from a population that was not large enough to perform a statistical analysis, the relationship between dynamic CT enhancement patterns and FDG-PET positivity 
is interesting. Previously, FDG-PET positivity was reported to be strongly associated with poorlydifferentiated HCC.[29] Therefore, FDG-PET positivity is usually a negative predictor of response to various treatments (i.e., surgical resection, TACE, and sorafenib).[24, 25, 30, 31] However, the results of the present study suggest that FDG-PET positivity along with a heterogeneous enhancement pattern appears to be relatively preferred.

Several years ago, Harimoto et al. reported that high FGFR2 expression is significantly correlated with poor histological differentiation, a higher incidence of portal vein invasion, and high AFP levels.[32] In contrast to sorafenib and regorafenib, lenvatinib inhibits FGFR1-4 in addition to VEGFR1-3, PDGFR-a, Ret, and Kit. The role of FGFR inhibition by lenvatinib may have influenced the results of the present study.

However, an insufficient amount of pre-clinical or clinical data regarding relationship estimation of tumor differentiation using dynamic CT enhancement pattern and treatment response to TKIs exists. These potential mechanisms need to be further investigated in future studies.

Figure 3 illustrates our proposed treatment strategy. For tumors with a homogeneous enhancement pattern, the early response rate to lenvatinib was high (ORR, 54\%). Therefore, for tumors that are unresectable and have failed or are refractory to TACE, administration of lenvatinib should be considered. In contrast, tumors with a heterogeneous enhancement pattern (Type-3 and -4) associated with a malignant gross type (SNEG and CMN) and that are histologically poorly differentiated[18] demonstrated a poor response to TACE[17, 33]. However, their response to lenvatinib was extremely high (ORR, 87-89\%). Therefore, when the tumor status is unresectable and the initial TACE response is insufficient, lenvatinib should be immediately administered.

FDG-PET/CT analysis is an optional imaging tool; even though it cannot be performed easily compared to other types of imaging analysis (i.e., CT or MRI) for various reasons (i.e., relatively high cost and a small number/uneven distribution of instruments), it may provide useful information for treatment selection. For tumors with a heterogeneous enhancement pattern, if the intrahepatic target nodules are PET-positive (TLR $\geq 2$ ), these target nodules may have high malignant potential and may rapidly progress. Although the study sample was very small and the data are immature, the treatment response was extremely high (ORR, 100\%). Therefore, for tumors that are unresectable, lenvatinib should be immediately administered. However, because this strategy was developed based on the results of our small retrospective analysis, a multicenter study enrolling a larger number of patients is required to verify this strategy.

The majority of the cases examined in this study are post-marketing cases, so long-term analysis has not yet been completed. However, after lenvatinib administration was initiated in one patient (BCLC stage C) in a global Phase III trial and in two patients (BCLC stage B and C, respectively) in the post-marketing period, a cancer-free state was achieved following additional treatment. In addition, two of these patients have remained cancer-free even after discontinuing lenvatinib (Figure 4). Compared to other TKIs, lenvatinib appears to decrease blood flow very rapidly, and as a result, evaluation of response to the 
agent can be done quickly. In both of the cases described above, lenvatinib played a role as a bridging therapy for surgical resection, with the aim of achieving a cancer-free state. Most importantly, no new lesions appeared within the treatment period. Even if new lesions appear after surgical resection, they could possibly be controlled following reinitiation of lenvatinib.

This study has some limitations. First, this was a retrospective, single-center, cohort study that evaluated a small number of patients. Second, the follow-up period was short compared to that of the global Phase III trial [16] (median follow-up period: REFLECT trial, 27.7 months; this retrospective study, 6.6 months); therefore, it is not possible yet to perform an adequate prognostic analysis. Finally, the diagnosis of HCC was based essentially on image analysis. A large-scale study is required to evaluate the utility of heterogeneous dynamic study enhancement patterns as biomarkers in the treatment of HCC by lenvatinib.

\section{Conclusions}

Lenvatinib resulted in a good early treatment response to unresectable HCC. In addition, estimation of tumor differentiation using image analysis was a useful method for predicting early tumor response.

\section{Abbreviations}

AEs, adverse events; AFP, alpha-fetoprotein; AST, aspartate aminotransferase; BCLC, Barcelona clinic liver cancer; CTCAE, Common Terminology Criteria for Adverse Events; CR, complete response; CT, computed tomography; CMN, confluent multi-nodular; DCP, des-y-carboxyprothrombin; FGFR, fibroblast growth factor receptor; FDG, fluorine-18-fluorodeoxyglucose; HBV, hepatitis B virus; HCV, hepatitis C virus; HCC, hepatocellular carcinoma; HU, Hounsfield units; mRECIST, Modified Response Evaluation Criteria in Solid Tumors; MDCT, multidetector CT; NonB,NonC, neither HBV nor HCV infection present; OR, objective response; ORR, objective response rate; $O S$, overall survival; $P R$, partial response; PDGRF, platelet-derived growth factor receptor; $\mathrm{PET}$, positron emission tomography; $\mathrm{PD}$, progressive disease; ROI, region-ofinterest; SNEG, simple nodular type with extranodular growth; SD, stable disease; TACE, transcatheter arterial chemo-embolization; TN, target nodule; TLR, tumor to liver ratio; TKI, tyrosine kinase inhibitor; VEGFR, vascular endothelial growth factor receptor and VOI, volume-of-interest .

\section{Declarations}

Funding: This work was supported by Japan's insurance system prescribed by the ministry of health, Labor and Welfare (No grant number). The Japan's Ministry of Health, Labor and Welfare had no role in the design of the study and collection, analysis, and interpretation of data and in writing the manuscript.

Ethics Approval and Consent to Participate: This retrospective non-intervention study was approved by the Institutional Review Board, Toranomon Hospital (protocol number; 1438-H/B). The study was performed in accordance with the Declaration of Helsinki. Because of the anonymous nature of the 
data and the opt-out disclosed on our institution's homepage (http://www.crc-

toranomonhosp.jp/document/clinical443.pdf), the requirement for additional informed consent to participate in this study was deemed unnecessary according to the Japanese national regulation "Ethical Guidelines for Medical and Health Research Involving Human Subjects" (https://www.mhlw.go.jp/file/06Seisakujouhou-10600000-Daijinkanboukouseikagakuka/0000080278.pdf).

Consent for publication: Not applicable.

\section{Competing Interests:}

Masahiro Kobayashi, M.D. reports honoraria from Eisai.

The other authors declare no conflicts of interest.

Availability of data and materials: The datasets used and/or analyzed during the current study are not available due to personal privacy.

Authorship: All authors had access to the data and participated in the writing of this manuscript.

Acknowledgement: Not applicable.

Author contributions: All authors contributed to the study design as well as collection and analysis. YK-1 (Yususke Kawamura) developed the study concept, performed statistical analysis, and drafted the manuscript. MK supervised the study design and performed statistical analysis. JS critically revised the manuscript. KI supervised the study design. YK-2 (Yuta Kobayashi), KK, TS, SF, TH, SS, HS, NA, FS, YS, YA, $\mathrm{MH}, \mathrm{HK}$ were involved in data assembly and interpretation. All of the authors read and approved the final manuscript.

\section{References}

1. Ferlay J, Soerjomataram I, Dikshit R, Eser S, Mathers C, Rebelo M, Parkin DM, Forman D, Bray F: Cancer incidence and mortality worldwide: sources, methods and major patterns in GLOBOCAN 2012. International journal of cancer 2015, 136(5):E359-386.

2. Forner A, Reig ME, de Lope CR, Bruix J: Current strategy for staging and treatment: the BCLC update and future prospects. Seminars in liver disease 2010, 30(1):61-74.

3. Forner A, Reig M, Bruix J: Hepatocellular carcinoma. The Lancet 2018, 391(10127):1301-1314.

4. Mazzaferro V, Llovet JM, Miceli R, Bhoori S, Schiavo M, Mariani L, Camerini T, Roayaie S, Schwartz $\mathrm{ME}, \mathrm{Grazi} \mathrm{GL}$ et al: Predicting survival after liver transplantation in patients with hepatocellular carcinoma beyond the Milan criteria: a retrospective, exploratory analysis. The Lancet Oncology 2009, 10(1):35-43.

5. Pugh RN, Murray-Lyon IM, Dawson JL, Pietroni MC, Williams R: Transection of the oesophagus for bleeding oesophageal varices. The British journal of surgery 1973, 60(8):646-649. 
6. Kawamura Y, Ikeda K, Hirakawa M, Hosaka T, Kobayashi M, Saitoh S, Yatsuji H, Sezaki H, Akuta N, Suzuki $\mathrm{F}$ et al: Efficacy of platinum analogue for advanced hepatocellular carcinoma unresponsive to transcatheter arterial chemoembolization with epirubicin. Hepatology research : the official journal of the Japan Society of Hepatology 2009, 39(4):346-354.

7. Seki A, Hori S: Switching the loaded agent from epirubicin to cisplatin: salvage transcatheter arterial chemoembolization with drug-eluting microspheres for unresectable hepatocellular carcinoma. Cardiovascular and interventional radiology 2012, 35(3):555-562.

8. Hiraoka A, Kumada T, Kudo M, Hirooka M, Koizumi Y, Hiasa Y, Tajiri K, Toyoda H, Tada T, Ochi H et al: Hepatic Function during Repeated TACE Procedures and Prognosis after Introducing Sorafenib in Patients with Unresectable Hepatocellular Carcinoma: Multicenter Analysis. Digestive diseases 2017, 35(6):602-610.

9. Llovet JM, Ricci S, Mazzaferro V, Hilgard P, Gane E, Blanc JF, de Oliveira AC, Santoro A, Raoul JL, Forner A et al: Sorafenib in advanced hepatocellular carcinoma. The New England journal of medicine 2008, 359(4):378-390.

10. Cheng AL, Kang YK, Chen Z, Tsao CJ, Qin S, Kim JS, Luo R, Feng J, Ye S, Yang TS et al: Efficacy and safety of sorafenib in patients in the Asia-Pacific region with advanced hepatocellular carcinoma: a phase III randomised, double-blind, placebo-controlled trial. The Lancet Oncology 2009, 10(1):25-34.

11. Ogasawara S, Chiba T, Ooka Y, Kanogawa N, Motoyama T, Suzuki E, Tawada A, Kanai F, Yoshikawa $\mathrm{M}$, Yokosuka O: Efficacy of sorafenib in intermediate-stage hepatocellular carcinoma patients refractory to transarterial chemoembolization. Oncology 2014, 87(6):330-341.

12. Arizumi T, Ueshima K, Minami T, Kono M, Chishina H, Takita M, Kitai S, Inoue T, Yada N, Hagiwara S et al: Effectiveness of Sorafenib in Patients with Transcatheter Arterial Chemoembolization (TACE) Refractory and Intermediate-Stage Hepatocellular Carcinoma. Liver Cancer 2015, 4(4):253-262.

13. Ohki T, Kondo M, Karasawa Y, Kawamura S, Maeshima S, Kojima K, Seki M, Toda N, Shioda Y, Tagawa K: Evaluation of the Efficacy of Sorafenib on Overall Survival in Patients with Hepatocellular Carcinoma using FT Rate: A Devised Index. Advances in therapy 2017, 34(5):1097-1108.

14. Markus Peck-Radosavljevic MK, Jean-Luc Raoul, Han Chu Lee, Thomas Decaens, Jeong Heo, ShiMing Lin, Hong Shan, Yefa Yang, Inga Bayh, Keiko Nakajima, Ann-Lii Cheng; Medical University of Vienna/ Klinikum Klagenfurt am Wörthersee, Vienna/Klagenfurt, Austria; Kindai University Faculty of Medicine, Osaka, Japan; Digestive Oncology, Institut de Cancérologie de l'Ouest, Nantes, France; Asan Medical Center, Seoul, Korea, Republic of (South); Clinique Universitaire d'Hépatogastroentérologie, CHU Grenoble Alpes, Grenoble, France; College of Medicine, Pusan National University and Medical Research Institute, Pusan National University Hospital, Busan, Korea, Republic of (South); Chang Gung Memorial Hospital, Taipei, Taiwan; Department of Radiology, The Third Affiliated Hospital of Sun Yat-Sen University, Guangzhou, Guangdong, China; Eastern Hepatobiliary Surgery Hospital, Second Military Medical University, Shanghai, China; Bayer AG, SBU Oncology, Pharmaceuticals, Wuppertal, Germany; Bayer HealthCare Pharmaceuticals, Whippany, NJ; National Taiwan University Hospital, Taipei, Taiwan: Outcomes of patients (pts) with hepatocellular 
carcinoma (HCC) treated with transarterial chemoembolization (TACE): Global OPTIMIS final analysis. J Clin Oncol 2018, 36:(suppl; abstr 4018).

15. Ikeda K, Kudo M, Kawazoe S, Osaki Y, Ikeda M, Okusaka T, Tamai T, Suzuki T, Hisai T, Hayato S et al: Phase 2 study of lenvatinib in patients with advanced hepatocellular carcinoma. Journal of gastroenterology 2017, 52(4):512-519.

16. Kudo M, Finn RS, Qin S, Han K-H, Ikeda K, Piscaglia F, Baron A, Park J-W, Han G, Jassem J et al: Lenvatinib versus sorafenib in first-line treatment of patients with unresectable hepatocellular carcinoma: a randomised phase 3 non-inferiority trial. The Lancet 2018, 391(10126):1163-1173.

17. Kanai T, Hirohashi S, Upton MP, Noguchi M, Kishi K, Makuuchi M, Yamasaki S, Hasegawa H, Takayasu K, Moriyama N et al: Pathology of small hepatocellular carcinoma. A proposal for a new gross classification. Cancer 1987, 60(4):810-819.

18. Kawamura Y, Ikeda K, Hirakawa M, Yatsuji H, Sezaki H, Hosaka T, Akuta N, Kobayashi M, Saitoh S, Suzuki F et al: New classification of dynamic computed tomography images predictive of malignant characteristics of hepatocellular carcinoma. Hepatology research : the official journal of the Japan Society of Hepatology 2010, 40(10):1006-1014.

19. Kawamura Y, Ikeda K, Seko Y, Hosaka T, Kobayashi M, Saitoh S, Kumada H: Heterogeneous type 4 enhancement of hepatocellular carcinoma on dynamic $C T$ is associated with tumor recurrence after radiofrequency ablation. AJR American journal of roentgenology 2011, 197(4):W665-673.

20. Kawamura Y, Ikeda K, Fujiyama S, Hosaka T, Kobayashi M, Saitoh S, Sezaki H, Akuta N, Suzuki F, Suzuki $Y$ et al: Potential of a no-touch pincer ablation procedure that uses a multipolar radiofrequency ablation system to prevent intrasubsegmental recurrence of small and single hepatocellular carcinomas. Hepatology research : the official journal of the Japan Society of Hepatology 2016.

21. Ronot M, Bouattour M, Wassermann J, Bruno O, Dreyer C, Larroque B, Castera L, Vilgrain V, Belghiti J, Raymond E et al: Alternative Response Criteria (Choi, European association for the study of the liver, and modified Response Evaluation Criteria in Solid Tumors [RECIST]) Versus RECIST 1.1 in patients with advanced hepatocellular carcinoma treated with sorafenib. Oncologist 2014, 19(4):394-402.

22. Sultana S, Awai K, Nakayama Y, Nakaura T, Liu D, Hatemura M, Funama Y, Morishita S, Yamashita Y: Hypervascular hepatocellular carcinomas: bolus tracking with a 40-detector CT scanner to time arterial phase imaging. Radiology 2007, 243(1):140-147.

23. Hyun SH, Eo JS, Lee JW, Choi JY, Lee KH, Na SJ, Hong IK, Oh JK, Chung YA, Song BI et al: Prognostic value of (18)F-fluorodeoxyglucose positron emission tomography/computed tomography in patients with Barcelona Clinic Liver Cancer stages 0 and A hepatocellular carcinomas: a multicenter retrospective cohort study. European journal of nuclear medicine and molecular imaging 2016, 43(9):1638-1645.

24. Kitamura K, Hatano E, Higashi T, Seo S, Nakamoto Y, Yamanaka K, lida T, Taura K, Yasuchika K, Uemoto S: Preoperative FDG-PET predicts recurrence patterns in hepatocellular carcinoma. Annals of surgical oncology 2012, 19(1):156-162. 
25. Hatano E, Ikai I, Higashi T, Teramukai S, Torizuka T, Saga T, Fujii H, Shimahara Y: Preoperative positron emission tomography with fluorine-18-fluorodeoxyglucose is predictive of prognosis in patients with hepatocellular carcinoma after resection. World journal of surgery 2006, 30(9):17361741.

26. National Cancer Institute, Division of Cancer Treated and Diagnosis. Cancer Therapy Evaluation Program. Adverse events/CTCAE. Available at: https:

//ctepcancergov/protocolDevelopment/electronic_applications/ctchtm\#-ctc_40 Accessed December 23, 2018.

27. Lencioni R, Llovet JM: Modified RECIST (mRECIST) assessment for hepatocellular carcinoma. Seminars in liver disease 2010, 30(1):52-60.

28. Kudo M, Matsui O, Izumi N, Kadoya M, Okusaka T, Miyayama S, Yamakado K, Tsuchiya K, Ueshima K, Hiraoka A et al: Transarterial chemoembolization failure/refractoriness: JSH-LCSGJ criteria 2014 update. Oncology 2014, 87 Suppl 1:22-31.

29. Seo S, Hatano E, Higashi T, Hara T, Tada M, Tamaki N, Iwaisako K, Ikai I, Uemoto S: Fluorine-18 fluorodeoxyglucose positron emission tomography predicts tumor differentiation, P-glycoprotein expression, and outcome after resection in hepatocellular carcinoma. Clin Cancer Res 2007, 13(2 Pt 1):427-433.

30. Song MJ, Bae SH, Lee SW, Song DS, Kim HY, Yoo le R, Choi JI, Lee YJ, Chun HJ, Lee HG et al: 18Ffluorodeoxyglucose $\mathrm{PET} / \mathrm{CT}$ predicts tumour progression after transarterial chemoembolization in hepatocellular carcinoma. European journal of nuclear medicine and molecular imaging 2013, 40(6):865-873.

31. Sung PS, Park HL, Yang K, Hwang S, Song MJ, Jang JW, Choi JY, Yoon SK, Yoo IR, Bae SH: (18)Ffluorodeoxyglucose uptake of hepatocellular carcinoma as a prognostic predictor in patients with sorafenib treatment. European journal of nuclear medicine and molecular imaging 2018, 45(3):384391.

32. Harimoto N, Taguchi K, Shirabe K, Adachi E, Sakaguchi Y, Toh Y, Okamura T, Kayashima H, Taketomi A, Maehara Y: The significance of fibroblast growth factor receptor 2 expression in differentiation of hepatocellular carcinoma. Oncology 2010, 78(5-6):361-368.

33. Yamashita Y, Matsukawa T, Arakawa A, Hatanaka Y, Urata J, Takahashi M: US-guided liver biopsy: predicting the effect of interventional treatment of hepatocellular carcinoma. Radiology 1995, 196(3):799-804.

\section{Tables}

Table 1. Clinical profiles and laboratory data of patients with HCC treated with lenvatinib.* 


\section{Patient characteristics and laboratory data}

Number of patients

Gender, males:females
37

$26: 11$

$74(46-91)$

$22.4(11.9-30.1)$

$23: 3: 11$

$120(48-280)$

Platelet count, ' $10^{3} / \mathrm{mL}$ (range) ${ }^{\dagger}$

$3.7(3.0-4.2)$

Albumin, g/dL (range) ${ }^{\dagger}$

$1.0(0.3-2.8)$

Total bilirubin, mg/dL (range) ${ }^{\dagger}$

$82.4(64.9-118.5)$

Prothrombin activity, \% (range) ${ }^{\dagger}$

39 (15-351)

AST, IU/L (range) ${ }^{\dagger}$

$127.4(0.8-17,264.3)$

$\mathrm{AFP}, \mathrm{mg} / \mathrm{L}$ (range) ${ }^{\dagger}$

$277.0(13-41,109)$

$\mathrm{DCP}, \mathrm{AU} / \mathrm{L}$ (range) ${ }^{\dagger}$

20 (54\%):17 (46\%)

Child-Pugh score 5:6, n (\%)

2 (5\%):16 (43\%):19 (51\%)
Initial dose of lenvatinib, mg/kg (range)

Treatment history of other TKI, n (\%)
$0.16(0.07-0.28)$

$4(11 \%)$

\section{Tumor characteristics}

Tumor diameter, mm (range) ${ }^{\dagger}$

Number of tumors, n (\%)

Macrovasular invasion, n (\%)

Extrahepatic metastasis, n (\%)

Up-to-7 In:Out, n (\%)
$31.0(11.0-92.2)$

$4(1-200)$

$6(16 \%)$

$14(38 \%)$ 


\section{Pretreatment dynamic study enhancement pattern (number and ratio)}

${ }^{\square} \mathrm{AFP}$, alpha-fetoprotein; BCLC, Barcelona clinic liver cancer; AST, aspartate aminotransferase; DCP, des-gcarboxyprothrombin; HBV, hepatitis B virus; HCC, hepatocellular carcinoma; HCV, hepatitis C virus; NonB,NonC, neither HBV nor HCV infection present; TACE, transcatheter arterial chemo-embolization; and TKI, tyrosine kinase inhibitor

${ }^{\dagger}$ Data expressed as median (range)

Table 2. Early treatment response evaluation using mRECIST 8-12 weeks after initiation of lenvatinib in all patients and by BCLC stage.*

\begin{tabular}{|c|c|c|c|c|c|c|c|}
\hline \multirow{3}{*}{ 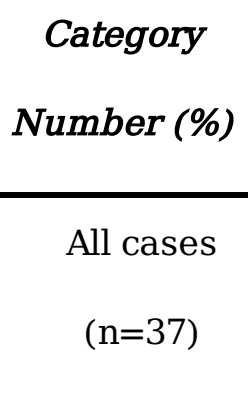 } & \multirow{2}{*}{$\begin{array}{c}\text { TACE } \\
\text { failure/refractoriness } \\
\text { Number (\%) } \\
33(89 \%)\end{array}$} & \multirow{2}{*}{$\begin{array}{c}\begin{array}{c}\text { Macrovascular } \\
\text { invasion }\end{array} \\
\text { Number (\%) } \\
6(16 \%)\end{array}$} & \multirow{2}{*}{$\begin{array}{c}\begin{array}{c}\text { Extrahepatic } \\
\text { spread }\end{array} \\
\text { Number (\%) } \\
14(38 \%)\end{array}$} & \multicolumn{4}{|c|}{$\begin{array}{c}\text { Response evaluation using } \\
\text { mRECIST } \\
\text { Number (\%) }\end{array}$} \\
\hline & & & & CR & PR & SD & PD \\
\hline & & & & $\begin{array}{c}6 \\
(16 \%)\end{array}$ & $\begin{array}{c}22 \\
(59 \%)\end{array}$ & $\begin{array}{c}6 \\
(16 \%)\end{array}$ & $\begin{array}{c}3 \\
(8 \%)\end{array}$ \\
\hline & & & \multicolumn{5}{|c|}{ OR } \\
\hline & & & \multicolumn{5}{|c|}{28 (76\%) } \\
\hline \multirow{4}{*}{$\begin{array}{r}\text { BCLC } \\
\text { stage A/B } \\
(n=21)\end{array}$} & 20 (95\%) & $0(0 \%)$ & $0(0 \%)$ & CR & PR & SD & PD \\
\hline & & & & $\begin{array}{c}4 \\
(19 \%)\end{array}$ & $\begin{array}{c}10 \\
(48 \%)\end{array}$ & $\begin{array}{c}4 \\
(19 \%)\end{array}$ & $\begin{array}{c}3 \\
(14 \%)\end{array}$ \\
\hline & & & \multicolumn{5}{|c|}{ OR } \\
\hline & & & \multicolumn{5}{|c|}{$14(67 \%)$} \\
\hline \multirow{4}{*}{$\begin{array}{c}\text { BCLC } \\
\text { stage C } \\
(n=16)\end{array}$} & 13 (81\%) & $6(38 \%)$ & 14 (88\%) & CR & PR & SD & PD \\
\hline & & & & $\begin{array}{c}2 \\
(13 \%)\end{array}$ & $\begin{array}{c}12 \\
(75 \%)\end{array}$ & $\begin{array}{c}2 \\
\text { (13\%) }\end{array}$ & $\begin{array}{c}0 \\
(0 \%)\end{array}$ \\
\hline & & & & \multicolumn{4}{|c|}{ OR } \\
\hline & & & & \multicolumn{4}{|c|}{14 (88\%) } \\
\hline
\end{tabular}

${ }^{\square}$ BCLC, Barcelona Clinic Liver Cancer; CR, complete response; mRECIST, Modified Response Evaluation Criteria in Solid Tumors; OR, objective response; PR, partial response; SD, stable disease; TACE, transcatheter arterial chemo-embolization; and $\mathrm{PD}$, progressive disease. 
Table 3. Evaluation of early treatment response after initiation of lenvatinib by dynamic CT enhancement pattern (Type-2 to -4 ) and analysis of imaging features.*

\begin{tabular}{|c|c|c|c|c|c|c|}
\hline \multirow{5}{*}{$\begin{array}{c}\text { Homogeneous } \\
\text { enhancement pattern }\end{array}$} & \multirow{5}{*}{$\begin{array}{c}\text { Dynamic CT image of main } \\
\text { target nodule Type and } \\
\text { Number }\end{array}$} & \multirow{3}{*}{$\begin{array}{c}\begin{array}{c}\text { Target nodule } \\
(T N)(H U) \text { and }\end{array} \\
\begin{array}{c}\text { tumor to liver } \\
\text { ratio (TLR) }{ }^{\dagger}\end{array} \\
\begin{array}{c}\text { Target } \\
\text { nodule } \\
(H U)\end{array} \\
\begin{array}{c}103.48 \\
(73.73- \\
131.18)\end{array}\end{array}$} & \multicolumn{4}{|c|}{$\begin{array}{c}\text { Response evaluation using } \\
\text { mRECIST }\end{array}$} \\
\hline & & & CR & PR & SD & $\mathrm{PD}$ \\
\hline & & & $\begin{array}{c}1 \\
(8 \%)\end{array}$ & $\begin{array}{c}6 \\
(46 \%)\end{array}$ & $\begin{array}{c}5 \\
(38 \%)\end{array}$ & $\begin{array}{c}1 \\
(8 \%)\end{array}$ \\
\hline & & $T L R$ & \multicolumn{2}{|c|}{ OR } & & \\
\hline & & $\begin{array}{c}1.49(1.12- \\
1.88)\end{array}$ & \multicolumn{2}{|c|}{7 (54\%) } & & \\
\hline \multirow[t]{8}{*}{$\begin{array}{c}\text { Heterogeneous } \\
\text { enhancement pattern }\end{array}$} & Type-3 $(\mathrm{n}=15)$ & $\begin{array}{c}\text { Target } \\
\text { nodule } \\
\text { (HU) }\end{array}$ & CR & PR & SD & PD \\
\hline & & $\begin{array}{c}90.78 \\
(64.86- \\
138.90)\end{array}$ & $\begin{array}{c}3 \\
(20 \%)\end{array}$ & $\begin{array}{c}10 \\
(67 \%)\end{array}$ & $\begin{array}{c}1 \\
(7 \%)\end{array}$ & $\begin{array}{c}1 \\
(7 \%)\end{array}$ \\
\hline & & $T L R$ & \multicolumn{2}{|c|}{ OR } & & \\
\hline & & $\begin{array}{c}1.45(1.05- \\
2.06) \\
\end{array}$ & \multicolumn{2}{|c|}{13 (87\%) } & & \\
\hline & \multirow[t]{4}{*}{ Type-4 $(\mathrm{n}=9)$} & $\begin{array}{c}\text { Target } \\
\text { nodule } \\
(H U)\end{array}$ & CR & PR & SD & PD \\
\hline & & $\begin{array}{c}67.67 \\
(51.81- \\
98.64)\end{array}$ & $\begin{array}{c}2 \\
(22 \%)\end{array}$ & $\begin{array}{c}6 \\
(67 \%)\end{array}$ & $\begin{array}{c}0 \\
(0 \%)\end{array}$ & $\begin{array}{c}1 \\
(11 \%)\end{array}$ \\
\hline & & $T L R$ & \multicolumn{2}{|c|}{ OR } & & \\
\hline & & $\begin{array}{c}1.00(0.90- \\
1.53)\end{array}$ & \multicolumn{2}{|c|}{8 (89\%) } & & \\
\hline
\end{tabular}

${ }^{\square} \mathrm{CR}$, complete response; CT, computed tomography; HU, Hounsfield units; mRECIST, Modified Response Evaluation Criteria in Solid Tumors; OR, objective response; PR, partial response; SD, stable disease; TLR, tumor to liver ratio; $\mathrm{TN}$, target nodule; and $\mathrm{PD}$, progressive disease.

${ }^{\dagger}$ Data expressed as median (range) 
Table 4. Predictors of early treatment response to lenvatinib.* 


\begin{tabular}{|c|c|c|c|c|c|}
\hline Variable & Category & HR $(95 \% \mathrm{CI})$ & $P$ & $\operatorname{HR}(95 \% \mathrm{CI})$ & $P$ \\
\hline \multirow[t]{2}{*}{ Gender } & 1: Male & 1 & & & \\
\hline & 2: Female & $0.42(0.09-2.00)$ & 0.274 & & \\
\hline \multirow[t]{2}{*}{ Age, years } & $1:<70$ & 1 & & & \\
\hline & $2: \geq 70$ & $0.14(0.02-1.31)$ & 0.086 & & \\
\hline \multirow[t]{2}{*}{ Body mass index, $\mathrm{kg} / \mathrm{m}^{2}$} & $1:<22$ & 1 & & & \\
\hline & $2: \geq 22$ & $0.90(0.18-4.40)$ & 0.896 & & \\
\hline \multirow[t]{2}{*}{ Others:HCV } & 1: Others & 1 & & & \\
\hline & 2: HCV & $0.38(0.07-2.17)$ & 0.277 & & \\
\hline \multirow[t]{2}{*}{ Platelet count, $\times 10^{4} / \mu \mathrm{L}$} & $1:<10$ & 1 & & & \\
\hline & $2: \geq 10$ & $2.00(0.43-9.42)$ & 0.381 & & \\
\hline \multirow[t]{2}{*}{ Albumin, g/dL } & $1:<3.5$ & 1 & & & \\
\hline & $2: \geq 3.5$ & 3.75 (0.78-17.99) & 0.099 & & \\
\hline \multirow[t]{2}{*}{ Total bilirubin, mg/dL } & $1:<1.0$ & 1 & & & \\
\hline & $2: \geq 1.0$ & $1.44(0.31-6.62)$ & 0.639 & & \\
\hline \multirow[t]{2}{*}{ Prothrombin activity, \% } & 1: <80 & 1 & & & \\
\hline & $2: \geq 80$ & $0.60(0.10-3.51)$ & 0.574 & & \\
\hline \multirow[t]{2}{*}{ AST, IU/L } & $1:<40$ & 1 & & & \\
\hline & $2: \geq 40$ & $1.25(0.28-5.65)$ & 0.772 & & \\
\hline \multirow[t]{2}{*}{$\mathrm{AFP}, \mathrm{mg} / \mathrm{L}$} & $1:<100$ & 1 & & & \\
\hline & $2: \geq 100$ & $0.80(0.18-3.62)$ & 0.772 & & \\
\hline \multirow[t]{2}{*}{$\mathrm{DCP}, \mathrm{AU} / \mathrm{L}$} & $1:<300$ & 1 & & & \\
\hline & $2: \geq 300$ & $1.08(0.24-4.90)$ & 0.917 & & \\
\hline \multirow[t]{2}{*}{ Tumor diameter, mm } & $1:<30$ & 1 & & & \\
\hline & $2: \geq 30$ & $0.58(0.12-2.78)$ & 0.493 & & \\
\hline \multirow[t]{2}{*}{ Tumor number } & $1:<4$ & 1 & & & \\
\hline & $2: \geq 4$ & $0.90(0.18-4.40)$ & 0.896 & & \\
\hline \multirow[t]{2}{*}{ Macrovascular invasion } & 1: No & 1 & & & \\
\hline & 2: Yes & $0.583(0.09-3.88)$ & 0.577 & & \\
\hline \multirow[t]{2}{*}{ Extra hepatic metastasis } & 1: No & 1 & & & \\
\hline & 2: Yes & $6.93(0.76-63.03)$ & 0.086 & & \\
\hline Heterogeneous enhancement pattern & 1: No & 1 & & 1 & \\
\hline
\end{tabular}




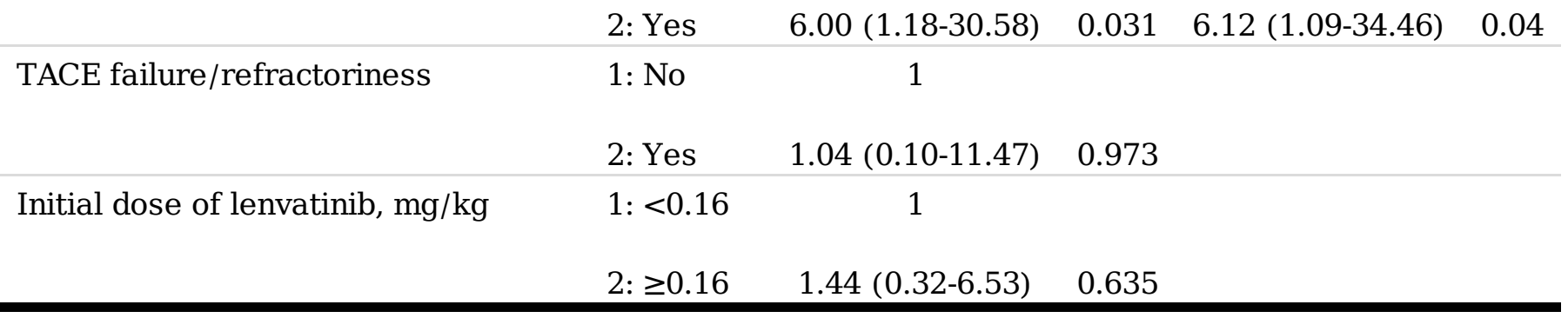

${ }^{\square}$ AFP, alpha-fetoprotein; AST, aspartate aminotransferase; CI, confidence interval; DCP, des-gcarboxyprothrombin; HCC, hepatocellular carcinoma; HCV, hepatitis C virus; HR, hazard ratio; and RFA, radiofrequency ablation.

Table 5. Relationship between dynamic CT enhancement patterns and tumor to liver ratio (TLR) using FDG-PET for early tumor response evaluation.*

\begin{tabular}{|c|c|c|c|c|c|c|c|}
\hline \multirow{2}{*}{$\begin{array}{c}\text { Category } \\
\text { Number (\%) } \\
\text { PET-negative }\end{array}$} & \multicolumn{3}{|c|}{$\begin{array}{l}\text { Distribution of dynamic CT enhancement pattern } \\
\text { Number (\%) }\end{array}$} & \multicolumn{4}{|c|}{$\begin{array}{c}\text { Response evaluation using mRECIST } \\
\text { Number (\%) }\end{array}$} \\
\hline & Туре-2 & Туре-3 & Туре-4 & CR & PR & SD & PD \\
\hline \multirow{3}{*}{$\begin{array}{l}\text { TLR }<2.0 \\
(n=13)\end{array}$} & & & & 1 (8\%) & $8(62 \%)$ & $3(23 \%)$ & $1(8 \%)$ \\
\hline & $8(62 \%)$ & 4 (31\%) & 1 (8\%) & \multicolumn{2}{|c|}{ OR } & & \\
\hline & & & & \multicolumn{2}{|c|}{$9(69 \%)$} & & \\
\hline PET-positive & Type-2 & Type-3 & Type-4 & CR & PR & SD & $\mathrm{PD}$ \\
\hline $\mathrm{TLR} \geq 2.0$ & & & & $2(50 \%)$ & $2(50 \%)$ & $0(0 \%)$ & $0(0 \%)$ \\
\hline \multirow{2}{*}{$(n=4)$} & $0(0 \%)$ & 1 (25\%) & 3 (75\%) & \multicolumn{2}{|c|}{ OR } & & \\
\hline & & & & \multicolumn{2}{|c|}{$4(100 \%)$} & & \\
\hline
\end{tabular}

${ }^{\square} \mathrm{CR}$, complete response; CT, computed tomography; FDG-PET, fluorodeoxyglucose-positron emission tomography; mRECIST, Modified Response Evaluation Criteria in Solid Tumors; OR, objective response; PR, partial response; SD, stable disease; TLR, tumor to liver ratio; and PD, progressive disease.

\section{Figures}




\section{Dynamic study, arterial phase}

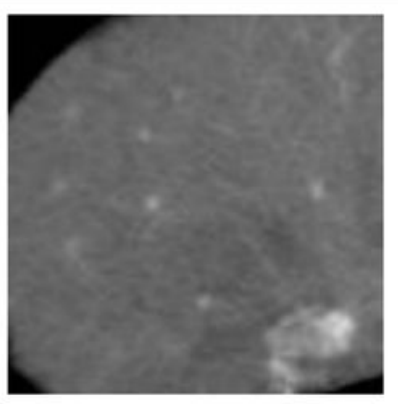

Type-1

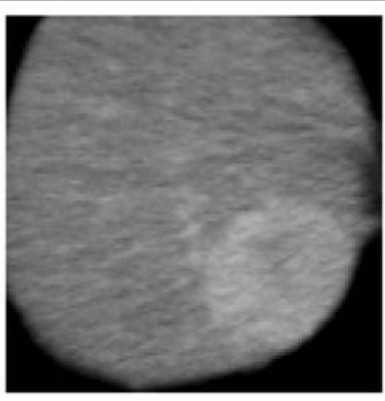

Type-2

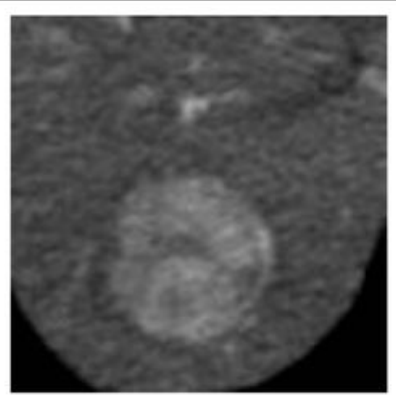

Type-3

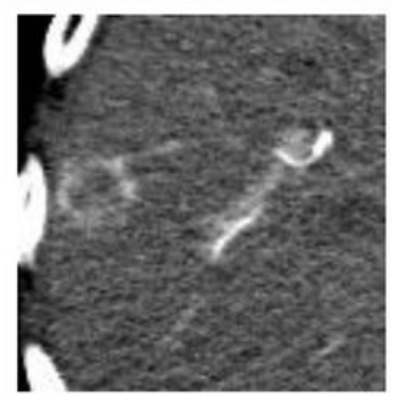

Type-4

\section{Dynamic study, portal phase}

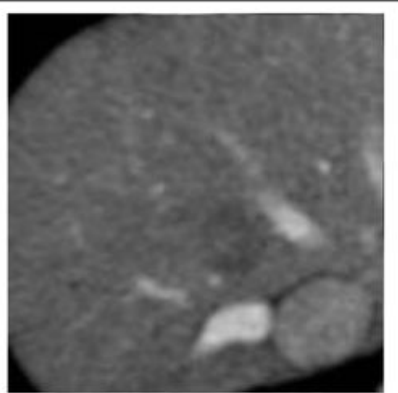

Type-1

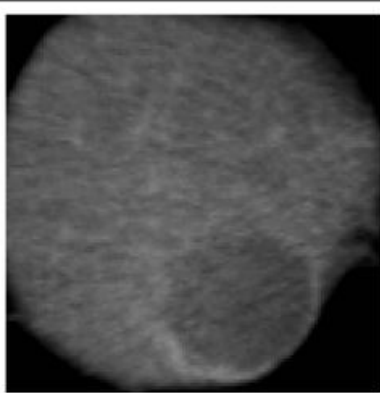

Type-2

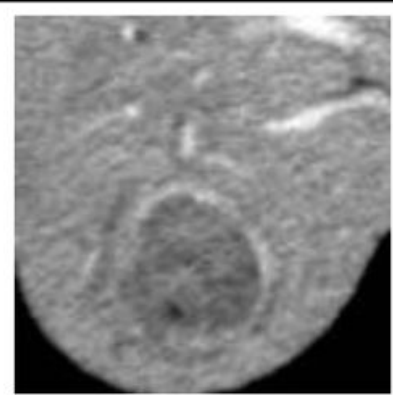

Type-3

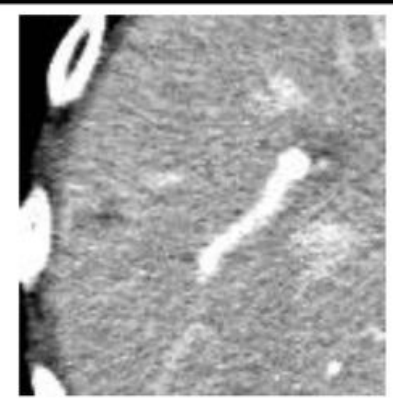

Type-4

(Reprinted with permission from John Wiley and Sons [20])

Figure 1

Original dynamic study images with each of the four enhancement patterns. (Reprinted with permission from John Wiley and Sons.[20]) 


\section{Arterial-phase dynamic CT images before and after initiation of}

lenvatinib in a patient with a heterogeneous Type-4 enhancement pattern
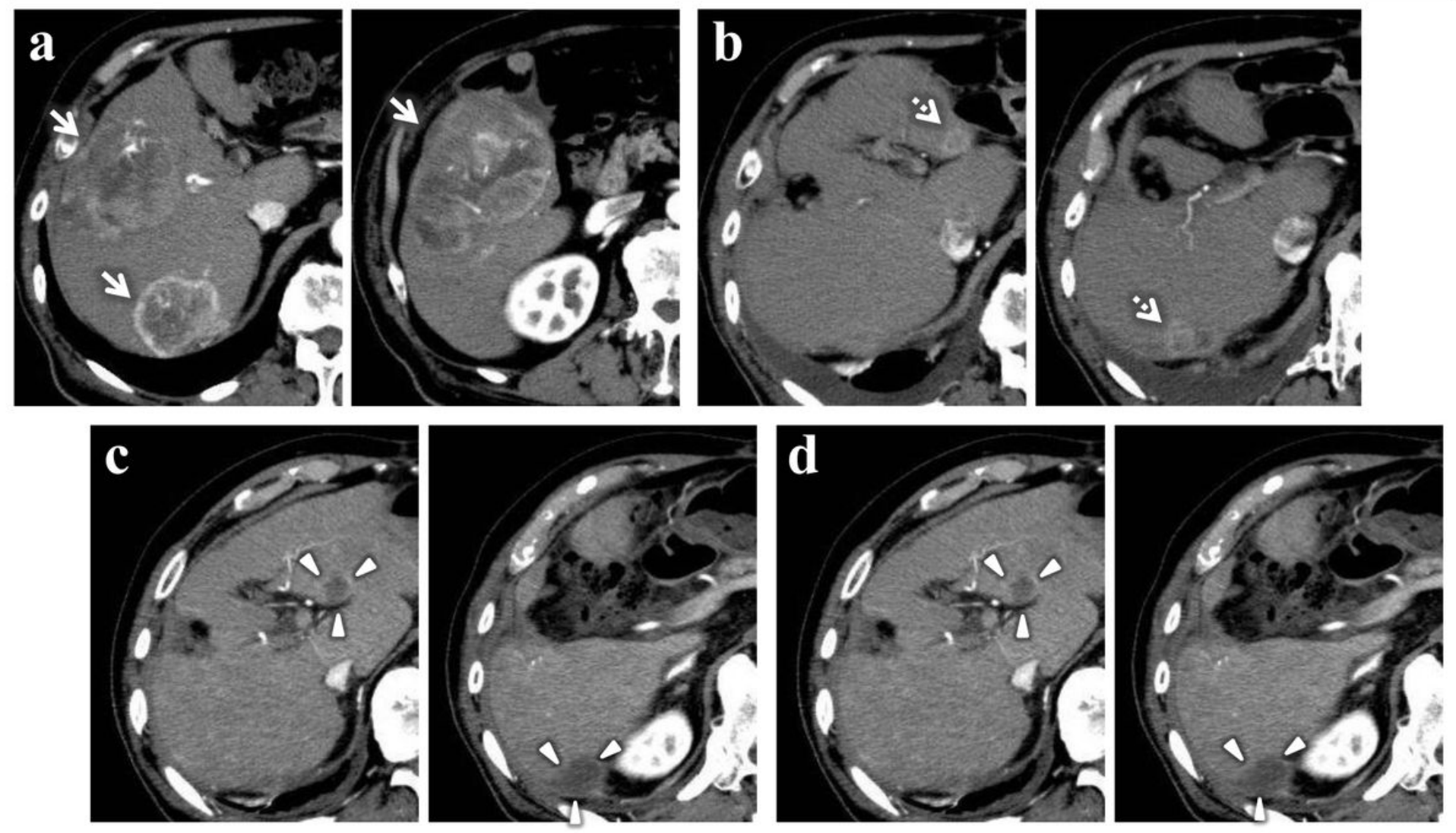

Figure 2

A male patient in his late 70s with alcoholic liver disease. Pretreatment dynamic CT images showing a Type-4 enhancement pattern (arrows) (a). Six months after surgical resection, multiple intrahepatic metastasis appeared (dotted arrows) and the pathological diagnosis was poorly-differentiated HCC with vp1 (b). Two weeks after initiation of lenvatinib for unresectable HCC, tumor vascularity dramatically decreased (arrowheads) (c). Moreover, decreasing tumor vascularity continued at 8 weeks after initiation of lenvatinib (arrowheads) (d). 


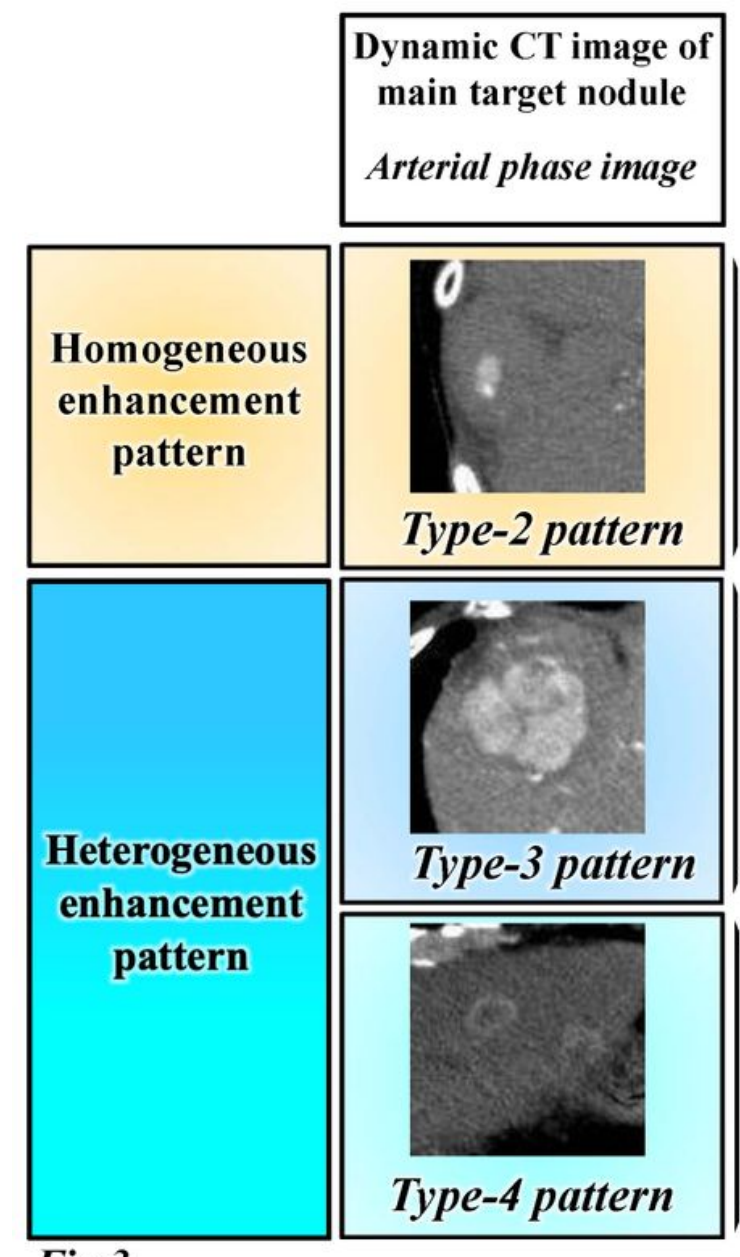

Fig.3
Early response rate to lenvatinib

${ }^{*}$ Rationale for timing of lenvatinib administration

\section{High}

*For tumors that are unresectable and failed or are refractory to TACE, lenvatinib administration should be considered

\section{Extremely} High

\section{*These heterogeneous} enhancement patterns may have high malignant potential

For tumors that are unresectable and the initial TACE response is insufficient, lenvatinib should be immediately administered
This strategy was developed based on results of our retrospective analysis.

Early response rate to lenvatinib according to TLR of main intrahepatic target nodules using FDG-PET/CT (optional analysis and additional information for considering timing of lenvatinib administration)

${ }^{\star}$ Rationale for timing of lenvatinib administration

\section{Early response rate may be high}

*PET-positive intrahepatic target nodules may indicate high malignant potential and may rapidly progress

PET-positive

TLR $\geq 2.0$

For tumors that are unresectable, lenvatinib should be immediately administered

\footnotetext{
*Treatment response was assessed using mRECIST
}

\section{Figure 3}

Strategy of lenvatinib administration for patients with HCC tumors that are unresectable and have failed or are refractory to TACE. 


\section{The potential of lenvatinib for converting advanced HCC patients to a resectable status}
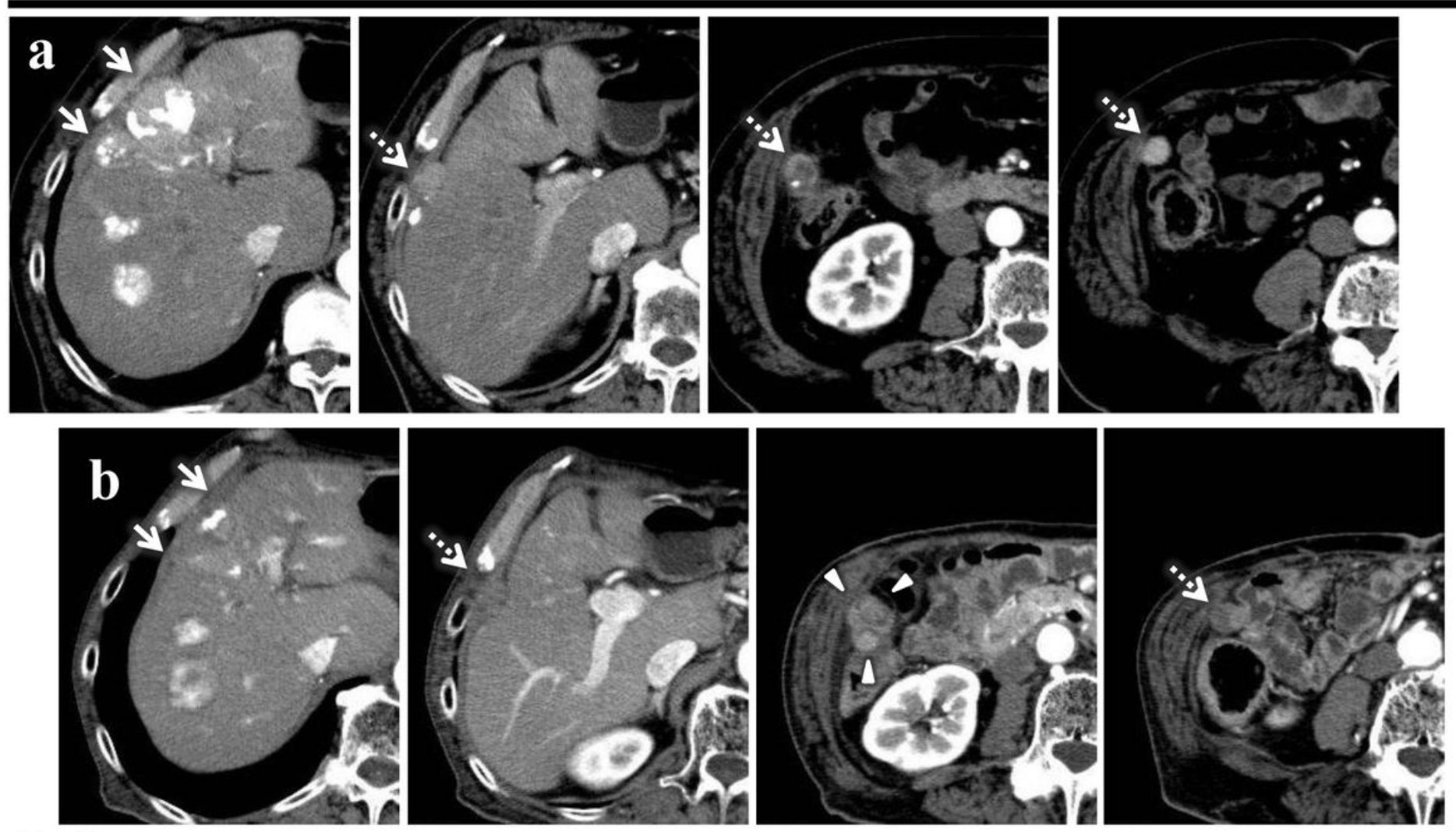

Fig. 4

Figure 4

A male patient in his early 60s with alcoholic liver disease. Pretreatment dynamic CT images showed an intrahepatic local tumor recurrence (arrows) and intraperitoneal tumor dissemination (dotted arrows) (a). About 3 years after initiation of lenvatinib for unresectable HCC, overall tumor control was good (arrows and dotted arrows), but one tumor in the intraperitoneal disseminated HCC increased in size (arrowheads). In this case, curative surgical resection was performed for intrahepatic and intraperitoneal disseminated HCC after stopping lenvatinib administration for almost 2 weeks. After surgical resection, he underwent resection and RFA for tumor recurrence, and has remained cancer-free without treatment for almost 19 months (b). 